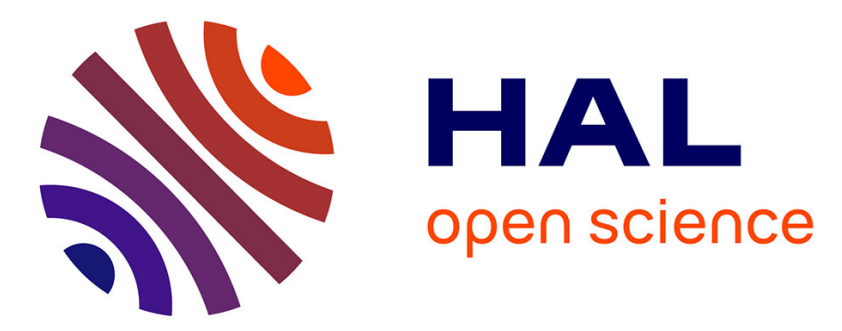

\title{
Lower and Upper Bounds for Minimum Energy Broadcast and Sensing Problems in Sensor Networks
}

Guillaume Chelius, Eric Fleury, Thierry Mignon

\section{To cite this version:}

Guillaume Chelius, Eric Fleury, Thierry Mignon. Lower and Upper Bounds for Minimum Energy Broadcast and Sensing Problems in Sensor Networks. [Research Report] RR-5072, INRIA. 2004. inria-00071511

\section{HAL Id: inria-00071511 \\ https://hal.inria.fr/inria-00071511}

Submitted on 23 May 2006

HAL is a multi-disciplinary open access archive for the deposit and dissemination of scientific research documents, whether they are published or not. The documents may come from teaching and research institutions in France or abroad, or from public or private research centers.
L'archive ouverte pluridisciplinaire HAL, est destinée au dépôt et à la diffusion de documents scientifiques de niveau recherche, publiés ou non, émanant des établissements d'enseignement et de recherche français ou étrangers, des laboratoires publics ou privés. 
INSTITUT NATIONAL DE RECHERCHE EN INFORMATIQUE ET EN AUTOMATIQUE

\title{
Lower and Upper Bounds for Minimum Energy Broadcast and Sensing Problems in Sensor Networks
}

\author{
Guillaume Chelius — Eric Fleury — Thierry Mignon
}

$\mathbf{N}^{\circ} \mathbf{5 0 7 2}$

January 2004

THÈME 1 



\title{
Lower and Upper Bounds for Minimum Energy Broadcast and Sensing Problems in Sensor Networks
}

\author{
Guillaume Chelius* , Eric Fleury* , Thierry Mignon ${ }^{\dagger}$ \\ Thème 1 - Réseaux et systèmes \\ Projet ARES \\ Rapport de recherche $\mathrm{n}^{\circ} 5072$ - January $2004-19$ pages
}

\begin{abstract}
In this document, we study the problem of assigning transmission and sensing ranges to the nodes of a wireless sensor network so as to minimize power consumption while ensuring broadcasting task or sensing process. A first novelty is that our model takes into account both the transmission and the reception costs when evaluating the energy consumption of a broadcasting task. We establish a new analytical model and derive lower and upper bounds on region covering. Moreover, we show that the lower bound is asymptotically optimal and can be approached up to $\epsilon$.
\end{abstract}

Key-words: Mathematical programming/optimization, Wireless, Sensor, Energy

Submitted for publication

* CITI/INSA de Lyon - ARES/INRIA

$\dagger$ UMR CNRS 5030 
Résumé : Ce rapport étudie le problème de l'affectation des puissances au sein d'un réseau de senseurs afin de minimiser l'énergie consommée par une opération de diffusion ou par les opérations de mesures. Un des apports majeurs de notre approche est qu'elle prend en compte à la fois le coût en émission et en réception. Nous établissons un modèle analytique et présentons des bornes inférieures et supérieures. De plus, nous montrons que ces bornes peuvent être approchées à $\epsilon$.

Mots-clés : Optimisation, énergie, réseau de capteurs, sans fil, diffusion 


\section{Introduction}

Sensor networks are made viable by the tremendous advances and convergence of microelectro-mechanical systems (MEMS), wireless communication technologies and digital electronics. Sensor networks are composed of a large number of tiny devices or sensors $[6,12,14]$. Each sensor contains an integrated sensor, a processor, a radio and an energy battery. Wireless sensor networks can be really useful in many civil and military areas for collecting, processing and monitoring environmental data $[1,15]$.

Sensor nodes are power constrained as each node operates with a limited energy budget. Moreover, since battery recharge is not always an available option, the lifetime of the entire network is a critical issue. Classical approaches such as low power hardware design provide individual solutions but remain insufficient for the energy constrained sensor systems. The energy consumption optimization of a sensor network is a really complex challenge. It involves a cut in the energy consumption of single sensor nodes but also requires global solutions in order to maximize the lifetime of the entire sensor network. The network lifetime can be increased through the introduction of energy aware mechanisms into each stage of a wireless sensor design, dissemination and operation.

There has been an increased awareness of energy efficient protocol need for batterypowered devices in recent years [9, 21, 23]. Though the optimization of the sensor network lifetime must take place at each stage, we focus mainly on the sensing and communication levels. Indeed, data transmission and reception using a wireless medium appears to be a highly energy consuming process. More precisely, our work is focused on a specific communication pattern called broadcast, where data is distributed from a source node to all other network nodes. Broadcast is a common and frequent process during a sensor network lifetime. It is useful for auto organization, parameter/data dissemination, or control regulation $[3,11,19]$. Conventional energy aware protocols manage the energy consumption by adjusting the transmission power of sensor nodes. Nodes are assumed to adapt their transmission power to the minimum required to sustain communication.

Several protocols have been proposed to adapt and manage the transmission ranges in sensor networks. All papers use and reference a simple energy model $[18,16,13]$. In this model, the amount of energy required to transmit data is proportional to the number of emitted bits and depends on both the communication range and the distance power gradient (see section 2). Note that regarding this model, reception of a message is not a low cost operation and can not be neglected in comparison to the transmission cost. Indeed, the amount of energy needed for a reception is in the same order of magnitude as the one needed for transmission and is also proportional to the number of received bits. In consequence, energy aware protocols should not only try to reduce communication ranges $[2,4,5,7,8$, $21,22,23]$ but should also minimize the number of transmission and reception operations for each message. In this research report, we look at the determination of minimum-cost (i.e., minimum energy consumption) broadcast and sensing schemes. Our main contribution is to take into account both the transmission and reception costs and to derive analytical bounds to the minimum energy broadcast and minimum energy sensing problems.

$\mathrm{RR} \mathrm{n}^{\circ} 5072$ 
The remaining of this report is structured as follow. In section 2, we present a model for communication and energy consumption in sensor networks. As opposed to the main previous works, we emphasis on the fact that the reception of a message is not a low cost operation. In section 3,4 and 6 , we derive upper and lower bounds for the minimum energy broadcast and sensing problems. We show some efficient covers and compute their energy consumption in 5 . We also show in 4 that any given region can be covered with an asymptotically optimal cover. Finally, section 7 summarizes our work and points out several future research directions.

\section{A discrete model}

The connectivity of a wireless network clearly depends on the transmission power of all hosts. We assume that each node is able to choose and tune its own power level, not to exceed some maximum value $\psi_{\max }$. It is generally and reasonably assumed in all previous works on the power assignment problem $[7,8,21,23]$ and stated in books on principles of wireless communication $[13,16]$ that the power $e(u)$ required by a host $u$ to correctly send data to another host $v$ satisfies the relation:

$$
\frac{e(u)}{d(u, v)^{\alpha}}>\gamma
$$

where $d(u, v)$ is the distance between $u$ an $v, \alpha \geq 1$ is the distance-power gradient, $\gamma \geq 1$ is the transmission-quality parameter. Note that in an "ideal" environment, $\alpha$ is generally equal to 2 but typically takes value between 2 and 4 , depending on the characteristics of the communication medium. Without loss of generality, we will assume trough all the paper that the threshold $\gamma$ (which depends on factors such as signal parameters, noise level...) is equal to 1.

\begin{tabular}{|c|c|}
\hline Radio mode & Power consumption (mW) \\
\hline Transmit & 14.88 \\
\hline Receive & 12.50 \\
\hline Idle & 12.36 \\
\hline Off & 0.016 \\
\hline
\end{tabular}

Table 1: Radio power characterization (taken in [17]).

We assume the use of omnidirectionnal antennas. Thus, due to the intrinsic broadcast nature of the wireless medium, all nodes that fall within the communication range of a given transmitting node $u$ are able to receive its transmission. This broadcast property of the wireless medium is called Wireless Multicast Advantage by [23]. It is clear that traditional models considering a "link based" approach are not relevant for wireless networks since links are not isolated anymore. Thus, when a node transmits, all its neighbors receive the signal. A general model will be 1 - port half duplex in reception (a host can only receive 
or transmit one message at a time) and $\Delta$-ports half duplex in emission (when sending, a host automatically sends its message towards all its neighbors) [10].

Keeping this transmission model in mind, we will model a wireless network using a directed graph $G=(V, E)$. Each node $u \in V$ is assigned a transmission power $\psi_{u} \leq \psi_{\text {max }}$. Each directed edge $e=(u, v) \in E$ is assigned a transmission cost $w_{u, v} \in R^{+}$equal to the minimum power needed to sustain the link $(u, v)$. We note $\Gamma(u)=\left\{v \in V \mid w_{u, v} \leq \psi_{\max }\right\}$ the neighbors of node $u$ (i.e., all nodes that fall into the maximum transmission range of node $u$ ). We note $C(u)=\left\{v \in V \mid w_{u, v} \leq \psi_{u}\right\}$ the covered of node $u$ (i.e., all nodes that fall into the transmission range $\psi_{u}$ of node $u$ ) and all nodes of $C(u)$ are said to be covered by node $u$. By definition, if node $u$ is transmitting at maximal power $\psi_{\max }$, all nodes of $\Gamma(u)$ are going to be covered by $u$.

As stated in the introduction, several papers $[13,18,16]$ points out the fact that receiving data via a wireless interface consumes a non negligible amount of energy. More precisely, this amount of energy consumed in reception is in the same order of magnitude as the amount of energy needed to send data (See Table 1). Thus, it appears really important to take into account both the transmitting cost and the receiving cost in a communication. In this report, one of the main goal is to study minimum energy broadcast trees. Given a root node $r$, the challenge is to find a set of relay nodes and a power assignment such that all nodes $v \in V$ are covered and such that the amount of consumed energy is minimum. A power assignment is a function $r$ :

$$
\begin{aligned}
r: V & \rightarrow\left[0, \psi_{\max }\right] \\
v \in V & \longmapsto \psi_{v} .
\end{aligned}
$$

If we note $\lambda$ the amount of energy consume in reception by one node, the total amount of energy consumed by the power assignment is:

$$
\sum_{u \in V} \psi_{v}+\sum_{u \in V} \lambda|C(u)|
$$

\section{Mathematical definitions}

In order to derive lower and upper bounds on the energy needed to cover a given area of the plane, we are first going to consider that we have a continuous density of sensors as in []. This mean that sensors are informally dispatched in the plane. model model is inpired from the works made in [20]. We also need to introduce some notations and definitions. In this whole report, if $P$ is a point of $\mathbb{R}^{2}$ and $r \in \mathbb{R}^{+}$, we call $D(P, r)$ the closed disk of center $P$ and radius $r$. We also call $A$ a subset of the real plane $\mathbb{R}^{2}$.

Definition $1 A$ cover of $A$, (also noted A cover), is a set of disks $\mathcal{R}=\left\{D\left(P_{i}, r_{i}\right), P_{i} \in\right.$ $\left.A, r_{i} \in \mathbb{R}^{+}, i \in I\right\}$, indexed by a countable set $I$, such that: 
1. The union of the disks of $\mathcal{R}$ contains $A$.

2. Any compact of the plane only meets a finite number of disks of $\mathcal{R}$.

The points $P_{i}$ are called the emitters of $\mathcal{R}$.

For the rest of this section, we fix a $A$ cover: $\mathcal{R}=\left\{D\left(P_{i}, r_{i}\right), i \in I\right\}$. If $P_{i}, P_{j}$ are two emitters of $\mathcal{R}$, we say that $P_{i}$ can transmit to $P_{j}$ if there is a sequence $i=i_{0}, i_{1}, \ldots, i_{r}=j$ of elements in $I$ verifying, for all $k=0, \ldots, r-1: P_{i_{k+1}} \in D\left(P_{i_{k}}, r_{i_{k}}\right)$.

Definition 2 We say that a cover $\mathcal{R}$ is:

- centralized if there is at least one emitter that can transmit to all emitters.

- connex if all emitters can transmit to all emitters.

\subsection{Constants and cost calculus}

Now, we fix three positive non-null constants, $\tau_{e}, \lambda$ and $\rho$, which are given by the model:

- $\tau_{e}$ is the relative emission cost, in $\mathrm{mW} / \mathrm{m}^{2}$.

- $\lambda$ is the reception cost consumed by a node (in $\mathrm{mW} /$ individual).

- $\rho$ is the number of nodes per square meter in the region $A$.

We deduce the two following constants:

- $\tau_{r}=\lambda . \rho$ is the relative reception cost, in $\mathrm{mW} / \mathrm{m}^{2}$.

- $\tau=\tau_{e}+\tau_{r}$ is the relative complete cost of the model, in $\mathrm{mW} / \mathrm{m}^{2}$.

Now, let $P_{i}$ be an emitter of $\mathcal{R}$; we can compute the following different costs considering $P_{i}$ :

- Emitting cost of $P_{i}=\tau_{e} \pi r_{i}^{2}$

- Reception cost induced by $P_{i}=\tau_{r} \pi r_{i}^{2}$

- Complete cost of $P_{i}=\tau \pi r_{i}^{2}$

Finally, for the entire cover $\mathcal{R}$, we fix the originate of the plane $O$ and define:

Definition 3 The absolute cost of a cover $\mathcal{R}$ is:

$$
\sum_{i \in I} \text { complete cost of emitter } P_{i}
$$

The relative cost of a cover $\mathcal{R}$ is:

$$
\limsup _{t \longrightarrow+\infty} \frac{\sum_{i \in I, P_{i} \in D(O, t)} \text { complete cost of emitter } P_{i}}{\operatorname{Area}(A \cap D(O, t))}
$$

INRIA 
Remark: The limit does not necessarily exist (it is easy to create covers such that there is no limit). This is why we have to consider the limit sup. In this report, and for problems addressed, covers we will consider have a relative regularity (see the examples in section 5) and they will admit a limit.

The absolute cost can be infinite if the region $A$ to cover is not bounded and if the emitters radius do not decrease fast enough. The relative cost can be infinite if, for example, the emitters radius (or the number of emitters) increase in a cubic manner as $t$ tends to infinity.

Remark: In this article, we suppose that the repartition of individuals is homogeneous. If it is not the case, we have to replace the constant $\rho$ by an integrable function $\rho: A \longrightarrow \mathbb{R}^{+}$. The reception cost of an emitter $P_{i}$ becomes $\int_{D\left(P_{i}, r_{i}\right)} \lambda \rho(x, y) d x d y$. Finally, the relative reception cost is replaced by a relative reception cost function $\tau_{r}=\lambda \rho$.

\section{Main results}

The aim of this mathematical study is the following:

Finding a centralized A cover with the smallest relative cost.

In the following, only the complete relative cost constant $\tau$ will be used. We fix a closed subset $A$ of $\mathbb{R}^{2}$.

\section{Theorem 1}

1. The relative cost of any $A$ cover is superior or equal to $\tau$.

2. For all $\varepsilon>0$, there is a connex $A$ cover whose relative cost is between $\tau$ and $\tau+\varepsilon$.

3. There is a connex cover of the plane whose relative cost is equal to $\tau$.

We will prove this theorem in section 6 . In the following section, we first present some examples of centralized, connex or basic plane covers.

\section{Cover examples}

All presented examples are periodical or semi-periodical covers such as defined below:

\subsection{Periodical covers}

Definition $4 A$ cover $\mathcal{R}$ indexed by $I$ is said to be periodical if there is a finite set $J \subset I$ and two vectors $u, v$ of $\mathbb{R}^{2}$ such that:

$$
\mathcal{R}=\cup_{(m, n) \in \mathbb{Z}^{2}}\left\{D\left(P_{j}+m u+n v, r_{j}\right), j \in J\right\}
$$

We say that $\mathcal{R}$ is the cover of period $\left\{D\left(P_{j}, r_{j}\right), j \in J\right\}$ generated by $u$ and $v$. 
Note that we do not assume that two disks $D_{i}, D_{j}$ indexed by different indexes are distinct. If this is the case, the cost of the two emitters is counted twice.

To compute the relative cost of a periodical cover, we can consider a compact $B \subset \mathbb{R}^{2}$ such that $\mathbb{R}^{2}=\cup_{(m, n) \in \mathbb{Z}^{2}}(B+m u+n v)$ and such that the interiors of $B$ and $B+m u+n v$ are disjoint for all $(m, n) \in Z^{2}$. (For example, we can choose $B$ to be the parallelogram of vertices $\{0, u, v, u+v\})$. Then, we have:

Proposition 1 The relative cost of $\mathcal{R}$ is:

$$
\frac{\sum_{j \in J} \pi r_{j}^{2}}{\operatorname{Area}(B)} \tau
$$

Proof : Let $b$ be the area of $B$. As $t$ tends to infinity, the number of $B$ translations contained in $\{D(O, t)\}$ is equal to $\frac{\pi t^{2}}{b}+O(t)$ (because $B$ exactly tiles $D(O, t)$ except for a stripe, at the disk edge, which area is $O(t))$. Since for all translated of $B$, there is exactly one copy of the cover period, the number of center of each period disks contained in $\{D(O, t)\}$ is also equal to: $\frac{\pi t^{2}}{b}+O(t)$. As a consequence, the relative cost is:

$$
\begin{aligned}
\text { Relative cost } & =\lim _{t \longrightarrow+\infty} \frac{\left(\frac{\pi t^{2}}{b}+O(t)\right) \cdot\left(\sum_{j \in J} \pi r_{j}^{2}\right)}{\pi t^{2}} \tau \\
& =\frac{\sum_{j \in J} \pi r_{j}^{2}}{\operatorname{Area}(B)} \tau
\end{aligned}
$$

\subsection{Semi-periodical covers}

Definition 5 Consider a periodical cover $\mathcal{R}$ of period $\left\{D_{1}, \ldots, D_{k}\right\}$ generated by $u$ and $v$. Consider a partition of $\mathbb{Z}^{2}$ in $r$ disjoint subsets: $\mathbb{Z}^{2}=Z_{1} \cup \cdots \cup Z_{r}$.

We can obtain a new cover by shifting each disk $D_{i}+m u+n v$ in a regular manner according to the membership of $(m, n)$ to one of the $Z_{i}$. Precisely, if we have kr vectors $w_{j, l}$, we give: $\forall j \in J,(m, n) \in \mathbb{Z}^{2}$, if $j \in J_{l}$ then $D_{j, m, n}^{\prime}=D_{j}+m u+n v+w_{j, l}$. We also define $\mathcal{R}^{\prime}=\left\{D_{j, m, n}^{\prime}, j, m, n \in J \times \mathbb{Z}^{2}\right\}$. If $\mathcal{R}^{\prime}$ is a plane cover, we say that it is semi-periodical of period $J$, generated by $u$ and $v$, and modified according to the partition $Z_{1}, \ldots, Z_{r}$ and the shifting vectors $w_{j, l}$.

Proposition 2 The relative cost of a semi-periodical cover is identical to the relative cost of any periodical cover the semi-periodical cover is derived from.

Proof : Translations do not change anything while passing to the limit 


\subsection{Square covers}

The covers that we call here "square covers" are semi-periodical covers generated by the canonical vectors $e_{1}=(1,0)$ and $e_{2}=(0,1)$. We give four examples in order to illustrate previous definitions.

\subsubsection{Simple square cover}

It is the periodical cover $\{D(O, \sqrt{2})\}$, generated by the canonical vectors $e_{1}=(1,0)$ and $e_{2}=(0,1)$, and depicted in figure 1 . It is not centralized. To compute its cost with formula (1), we consider the square $B$ of vertices $\{( \pm 1, \pm 1)\}$ and compute:

$$
\operatorname{cost}\left(\mathcal{R}_{1}\right)=\frac{\pi(\sqrt{2})^{2}}{2^{2}} \tau=\frac{\pi}{2} \tau \simeq 1,571 \tau .
$$

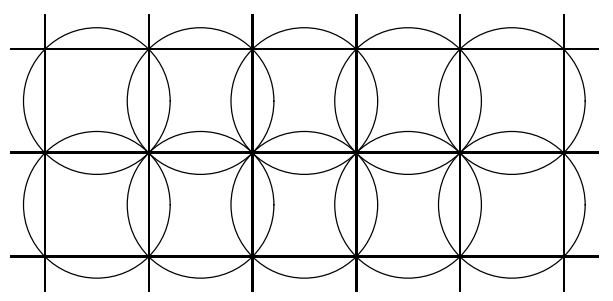

Figure 1: Simple square cover $(\operatorname{cost} \simeq 1,571 \tau)$.

\subsubsection{Connex square cover}

We increase the radius of the precedent cover such that all disks contain the nearest emitters. We get cover $\mathcal{R}_{2}$, of period $\{D(O, 2)\}$, generated by the canonical vectors $e_{1}=(1,0)$ and $e_{2}=(0,1)$, and depicted in figure 2 . To compute its cost, we consider the same square $B$ as for $\mathcal{R}_{1}$ :

$$
\operatorname{cost}\left(\mathcal{R}_{2}\right)=\frac{4 \pi 4}{4} \tau=\pi \tau \simeq 3,142 \tau
$$

\subsubsection{Centralized square cover I}

The first cover of this type is the semi-periodical cover presented in figure 3 . It is still generated by vectors $e_{1}$ and $e_{2}$, and the period we consider is: $\left\{D_{1}=D(O, \sqrt{2}), D_{2}=\right.$ 


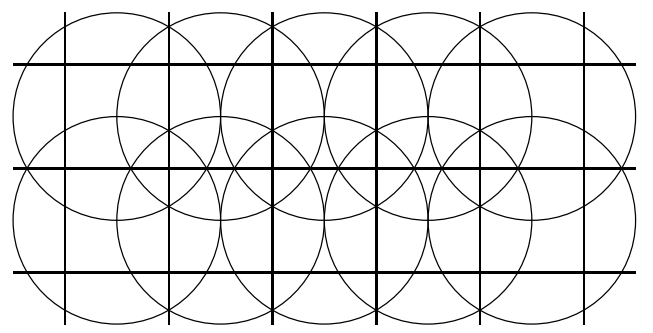

Figure 2: Connex square cover $($ cost $\simeq 3,142 \tau)$.

$D(O, 2-\sqrt{2})\}$. The partition of $\mathbb{Z}^{2}$ is $Z_{1} \cup Z_{2} \cup Z_{3} \cup Z_{4}$ with

$$
\begin{aligned}
(m, n) & \in Z_{1} \text { if } n>0 \\
& \in Z_{2} \text { if } n<0 \\
& \in Z_{3} \text { if } n=0 \text { and } m \geq 0 \\
& \in Z_{4} \text { if } n=0 \text { and } m<0
\end{aligned}
$$

In each of these zones, the big disk $D_{1}$ does not move, while the small disk $D_{2}$ is shifted on the bottom, on the top, on the left or on the right in order to allow the emitter in the middle of a square to transmit to the emitter in the middle of the following square. We do not present here the shifting vectors which can be easily computed.

The result is a centralized non-connex cover $\mathcal{R}_{3}$ which cost is computed using formula (1):

$$
\begin{aligned}
\operatorname{cost}\left(\mathcal{R}_{3}\right) & =\frac{\pi \sqrt{2}^{2}+\pi(2-\sqrt{2})^{2}}{4} \tau=(2-\sqrt{2}) \pi \tau \\
& \simeq 1,84 \tau .
\end{aligned}
$$

\subsubsection{Centralized square cover II}

In the previous cover, transmission between the square centers is provided by the presence of small circles of radius $2-\sqrt{2}$. By using twice more small circles and dividing their radius by 2 , we can ensure transmission at a lower cost. We get cover $\mathcal{R}_{4}$ given in figure 4 .

It is a semi-periodical cover that is derived from the periodical cover generated by the canonical vectors and the following period: $\left\{D_{1}=D(O, \sqrt{2}), D_{2}=D\left(O, \frac{2-\sqrt{2}}{2}\right), D_{3}=\right.$ $\left.D\left(O, \frac{2-\sqrt{2}}{2}\right)\right\}$. Its cost is:

$$
\begin{aligned}
\operatorname{cost}\left(\mathcal{R}_{4}\right) & =\frac{\pi \cdot \sqrt{2}^{2}+2 \pi\left(\frac{2-\sqrt{2}}{2}\right)^{2}}{4} \tau=\frac{7-2 \sqrt{2}}{8} \pi \tau \\
& \simeq 1,638 \tau .
\end{aligned}
$$




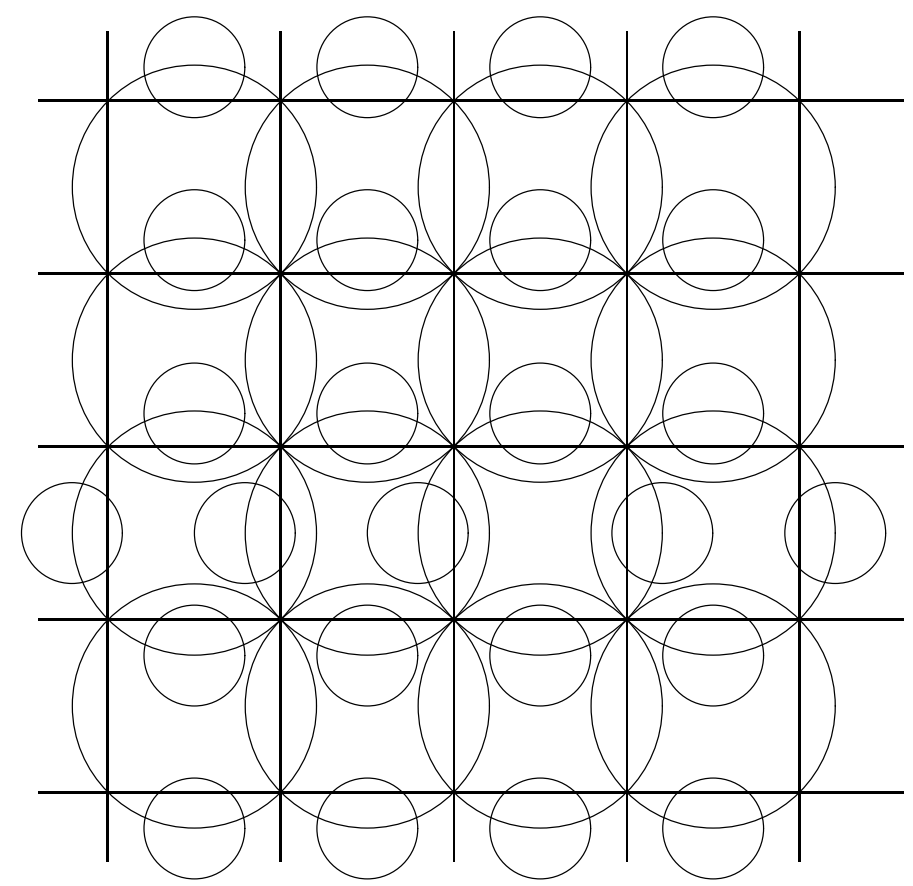

Figure 3: Centralized square cover I $(\operatorname{cost} \simeq 1,84 \tau)$

\subsection{Hexagonal covers}

Hexagonal covers are semi-periodical covers generated by vectors $u=\left(\frac{3}{2}, \frac{\sqrt{3}}{2}\right)$ and $v=$ $(0, \sqrt{3})$.

Let us call $B$ the hexagon of center $(0,0)$ which six vertices are $(1,0),(-1,0),\left( \pm \frac{1}{2}, \pm \frac{\sqrt{3}}{2}\right)$. The area of $B$ is $\frac{3 \sqrt{3}}{2}$ and the translations of $B$ by $\mathbb{Z} u+\mathbb{Z} v$ tile the plane.

\subsubsection{Simple hexagonal cover}

It is cover $\mathcal{R}_{5}$ of period $\{D(O, 1)\}$ generated by the group $\mathbb{Z} u+\mathbb{Z} v$ where $u$ and $v$ are defined as above. It is depicted in figure 5 .

Based on formula (1), its cost is:

$$
\operatorname{cost}\left(\mathcal{R}_{5}\right)=\frac{\pi 1^{2}}{\frac{3 \sqrt{3}}{2}} \tau=\frac{2 \pi}{3 \sqrt{3}} \tau \simeq 1,210 \tau
$$




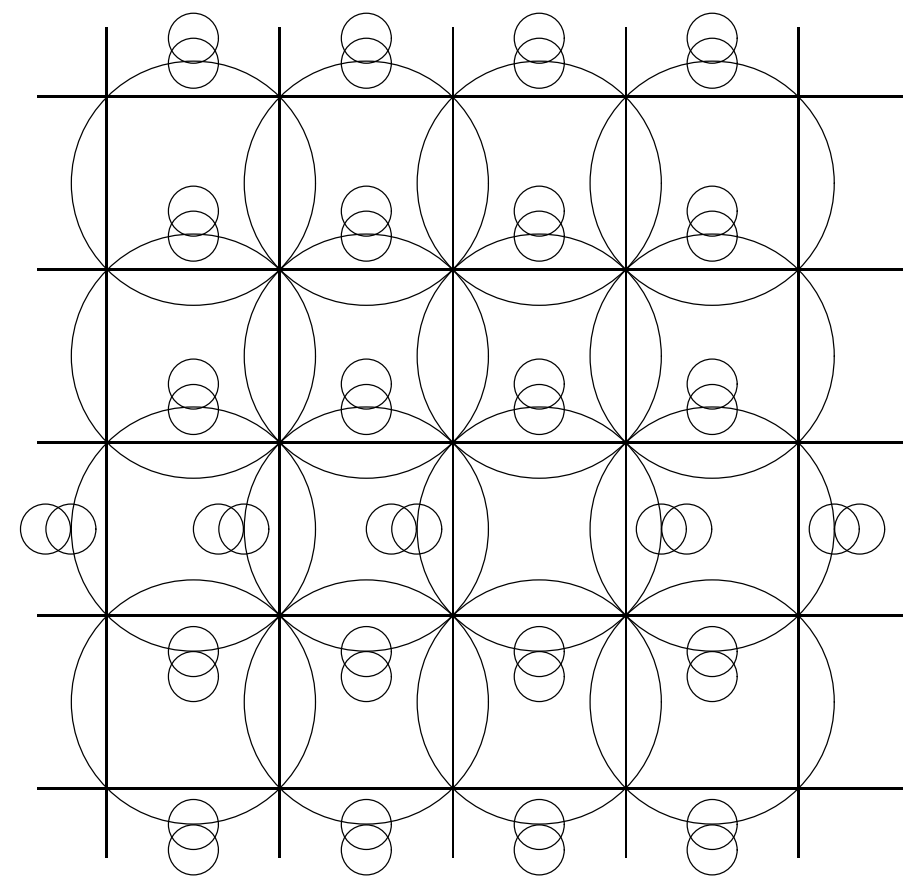

Figure 4: Centralized square cover II $($ cost $\simeq 1,638 \tau)$

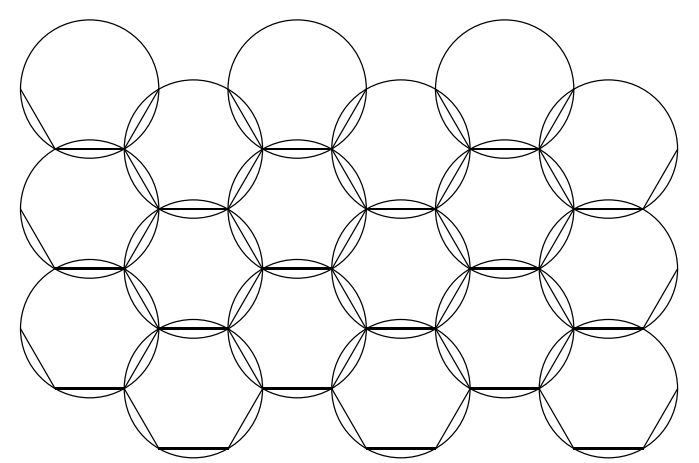

Figure 5: Simple hexagonal cover $($ cost $\simeq 1,210 \tau)$ 


\subsubsection{Connex hexagonal cover}

To construct a connex cover, we could, as for the connex square cover, increase the radius of the previous cover. We would have to consider a radius of $\sqrt{3}$ instead of 1 , and the cost of the cover would be $\frac{2}{\sqrt{3}} \pi \tau \simeq 3,628 \tau$.

Instead, we can choose to keep the same radius but to move the circle to the hexagon vertices.

We get the periodical cover $\mathcal{R}_{6}$ depicted in figure 6 . Its period is $\left\{D((1,0), 1), D\left(\left(\frac{1}{2}, \frac{\sqrt{3}}{2}\right), 1\right)\right\}$, it is generated by $u$ and $v$, and its cost is twice the one of cover $\mathcal{R}_{5}$, i.e.:

$$
\operatorname{cost}\left(\mathcal{R}_{6}\right)=\frac{4 \pi}{3 \sqrt{3}} \tau \simeq 2,418 \tau
$$

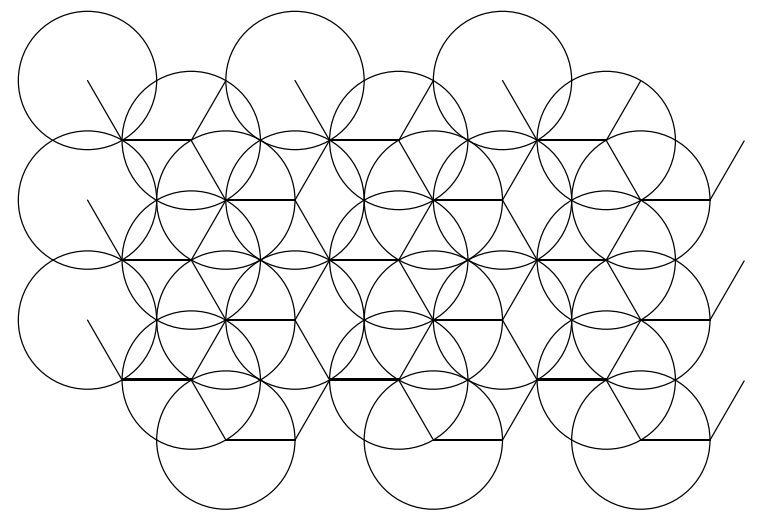

Figure 6: Connex hexagonal cover $(\operatorname{cost} \simeq 2,418 \tau)$

\subsubsection{Centralized hexagonal cover}

It is cover $\mathcal{R}_{7}$ given in figure 7 . It is semi-periodical and derived from the periodical cover generated by vectors $u, v$ and the following period: $\left\{D_{1}=D(O, 1), D_{2}=D\left(O, \frac{\sqrt{3}-1}{2}\right), D_{3}=\right.$ $D\left(O, \frac{\sqrt{3}-1}{2}\right\}$.

Its cost is:

$$
\begin{aligned}
\operatorname{cost}\left(\mathcal{R}_{7}\right) & =\frac{\pi 1^{2}+2 \pi\left(\frac{\sqrt{3}-1}{2}\right)^{2}}{\frac{3 \sqrt{3}}{2}}=\frac{6-2 \sqrt{3}}{3 \sqrt{3}} \pi \tau \\
& \simeq 1,533 \tau .
\end{aligned}
$$




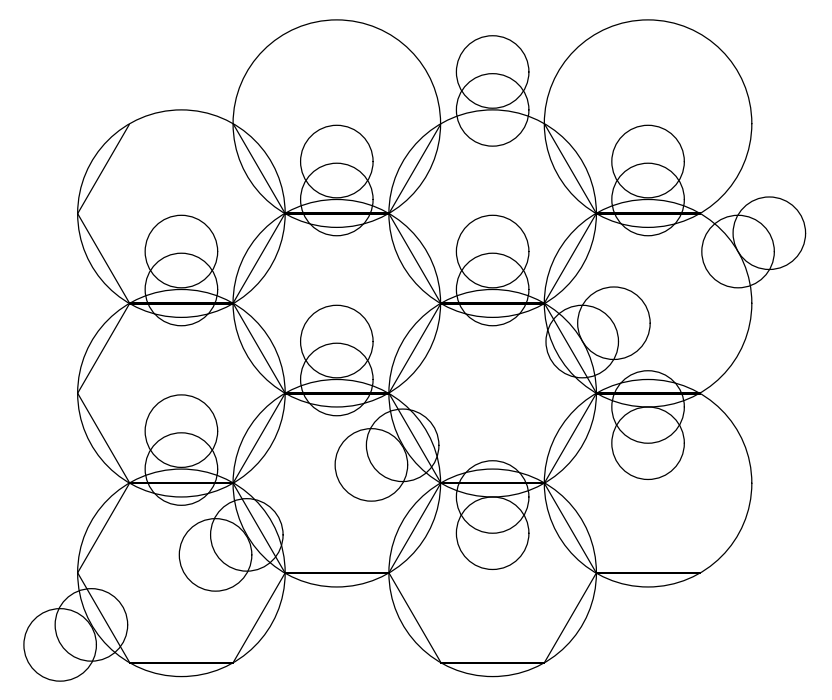

Figure 7: Centralized hexagonal cover $(\operatorname{cost} \simeq 1,533 \tau)$.

\section{Theorem's proof}

Examples presented above show that, starting from a non-centralized cover, we can construct a centralized one by adding "small circles" to connect emitters between each other. This is the process that was used to derive covers $\mathcal{R}_{3}$ and $\mathcal{R}_{4}$ from cover $\mathcal{R}_{1}$, or to construct cover $\mathcal{R}_{7}$ from cover $\mathcal{R}_{5}$. Moreover, reducing the small circles radius and increasing their number reduce the cover cost as shown in the transition from cover $\mathcal{R}_{3}$ to cover $\mathcal{R}_{4}$. This main idea leads the theorem's proof.

Definition 6 Let $\mathcal{R}$ be a cover (non centralized) of a region $A$. We call link for a cover $\mathcal{R}$ any set of disks $\mathcal{L}$ such that $\mathcal{R} \cup \mathcal{L}$ is a centralized cover of $A$.

Lemma 3 Let $\mathcal{R}$ be a cover of $A$, with a relative cost $\gamma$, and $\varepsilon$ a non-null positive real. There is a link $\mathcal{L}$ for $\mathcal{R}$ such that: $\operatorname{cost}(\mathcal{R} \cup \mathcal{L})<\gamma+\varepsilon$.

Proof : First consider two emitters $P$ and $Q$ at distance $d$ of each other. We can suppose that $P=(0,0)$ and $Q=(d, 0)$. Let $n \geq 2$ be an integer. We can connect $P$ and $Q$ using $n$ circles of radius $\frac{d}{n-1}$ centered in

$$
(0,0),\left(\frac{d}{n-1}, 0\right), \ldots,\left(\frac{k d}{n-1}, 0\right), \ldots,(d, 0) .
$$

The additional cost (absolute) of these circles is $n \pi\left(\frac{d}{n-1}\right)^{2} \tau$. When $n$ tends to infinity, the additional cost becomes as small as desired. 
Now consider the disk $D_{n}$ of radius $n$ centered at the origin. The number of emitters of $\mathcal{R}$ contained in $D_{1}$ is finite. In consequence, the method explained above allows us to connect all these emitters using a set of disks $\mathcal{L}_{1}$ which a total added cost inferior to $\frac{\varepsilon}{2} \operatorname{Area}\left(D_{1} \cap A\right)$.

Similarly, we can connect all emitters of $\mathcal{R} \cup \mathcal{L}_{1}$ contained in $D_{2}$ by a set of disks $\mathcal{L}_{2}$ with a total cost inferior to $\frac{\varepsilon}{2^{2}} \operatorname{Area}\left(D_{2} \cap A\right)$.

This way, we build a succession of sets $\mathcal{L}_{n}$ such that, for all $n$, emitters of $\mathcal{R} \cup \mathcal{L}_{1} \cup \cdots \cup \mathcal{L}_{n}$ contained in $D_{n}$ are connected, and such that the cost of $\mathcal{L}_{n}$ is inferior to $\frac{\varepsilon}{2^{n}} \operatorname{Area}\left(D_{n} \cap A\right)$.

Then, we set $\mathcal{L}=\mathcal{L}_{1} \cup \mathcal{L}_{2} \cup \cdots \cup \mathcal{L}_{n}$. The relative cost of $\mathcal{L}$ is equal to:

$$
\begin{aligned}
\operatorname{cost}(\mathcal{R} \cup \mathcal{L}) & \leq \operatorname{cost}(\mathcal{R})+\operatorname{cost}(\mathcal{L}) \\
& =\operatorname{cost}(\mathcal{R})+\limsup _{n \longrightarrow+\infty} \frac{\sum_{i=1}^{n} \operatorname{cost}\left(\mathcal{L}_{i}\right)}{\operatorname{Area}\left(D_{n} \cap A\right)} \\
& \leq \gamma+\limsup _{n \longrightarrow+\infty} \frac{\sum_{i=1}^{n} \frac{\varepsilon}{2^{i}} \operatorname{Area}\left(D_{i} \cap A\right)}{\operatorname{Area}\left(D_{n} \cap A\right)} \\
& \leq \gamma+\limsup _{n \longrightarrow+\infty} \varepsilon\left(\sum_{i=1}^{n} \frac{1}{2^{i}}\right)=\gamma+\varepsilon .
\end{aligned}
$$

By construction, the cover $\mathcal{R} \cup \mathcal{L}$ is connex.

\subsection{Theorem when $A$ is a square}

The first point of the theorem is obvious since the whole region $A$ is covered by disks of $\mathcal{R}$. For the second point, we first suppose that $A$ is the square $[-1,1] \times[-1,1]$. We give two lemmas:

Lemma 4 There is a sequence of disks $D_{n}$ contained in $A$, which interiors are pairwise disjoint and such that $\lim _{n \longrightarrow+\infty} \operatorname{Area}\left(D_{n}\right)=\operatorname{Area}(A)$.

Proof : Let us fix $D_{1}=D(O, 1)$, the unique disk tangent to the four sides of $A$. In every corner of $A$, there is an uncovered region bounded by three arcs: two sides of the square (which are circles of infinite radius), and the boundary of $D_{1}$. These three circles are tangent to each others, and we take $D_{2}$ to be the Soddy disk associated to these three circles (i.e. the unique disk tangent to these three circles). This partly fill the corner, but creates three new (smaller) uncovered regions still in the corner, each of them being limited by three tangent circles. We can repeat the process, partly filling each new region by a Soddy circle (in the four corners). It is known that the union of all the Soddy circles has the same area as the square and -by construction- their interiors are pairwise disjoint.

Lemma 5 Let $B \subset \mathbb{R}^{2}$ be a compact. For each $\eta>0$ there is a cover $\mathcal{R}(\eta)$ of $B$ such that

$$
\operatorname{Cost}(\mathcal{R}(\eta)) \leq \alpha \tau \operatorname{Area}(B)+e(\eta)
$$

where $\alpha=\frac{2 \pi}{3 \sqrt{3}}$ is the density of the simple hexagonal cover and $\lim _{\eta \rightarrow 0} e(\eta)=0$. 
Proof : Let $\mathcal{R}^{\prime}(\eta)=\left\{D_{l}, l \in L\right\}$ be the hexagonal cover of $\mathbb{R}^{2}$ by disks of diameter $\eta$. The relative cost of $\mathcal{R}^{\prime}(\eta)$ is $\alpha \tau$. We fix now:

$$
\mathcal{R}(\eta)=\left\{D_{l}, l \in L, D(l) \cap B \neq \emptyset\right\} .
$$

The set $\mathcal{R}(\eta)$ is a cover of $B$ contained in the set

$$
B(\eta)=\left\{x \in \mathbb{R}^{2}, \operatorname{distance}(x, B) \leq \eta\right\},
$$

and, then, its cost is less than $\alpha \tau \operatorname{Area}(B(\eta))$. Since $\operatorname{Area}(B(\eta))$ tends to Area $(B)$ as $\eta$ tends to zero, this concludes the proof of the lemma.

We come now to the proof of the theorem, an $\varepsilon$ being fixed. Consider the sequence of disks $D_{n}$ as in lemma 4 and note $a_{n}=4-\operatorname{Area}\left(D_{1} \cup \cdots \cup D_{n}\right)$ (the area of the uncovered region of $A$ ). Take $n \in \mathbb{N}$ such that $a_{n} \leq \frac{3 \varepsilon}{\tau \alpha}$.

Fix $B=\overline{A-\cup_{i=1}^{n} D_{i}}$ and choose a cover $\mathcal{R}(\eta)$ of $B$ as in lemma 5 above such that $\operatorname{Cost}(\mathcal{R}(\eta)) \leq \alpha \tau \operatorname{Area}(B)+\varepsilon$.

Fix the cover $\mathcal{P}=\mathcal{R}(\eta) \cup\left(\cup_{i=1}^{n} D_{i}\right)$. We have:

$$
\begin{aligned}
\operatorname{Cost}(\mathcal{P}) & =\operatorname{Cost}(\mathcal{R})+\operatorname{Cost}\left(\cup_{i=1}^{n} D_{i}\right) \\
& \leq \alpha \tau \operatorname{Area}(B)+\varepsilon+\tau \operatorname{Area}\left(\cup_{i=1}^{n} D_{i}\right) \\
& =\alpha \tau a_{n}+\varepsilon+\tau\left(4-a_{n}\right) \\
& \leq 4 \varepsilon+4 \tau .
\end{aligned}
$$

It follows that the relative cost of $\mathcal{P}$ is less than $\tau+\varepsilon$, which concludes the proof.

\subsection{Theorem's sketch of proof for the remaining points}

\subsubsection{If the region $A$ is not a square}

Fix a (small) $\eta>0$ and consider a plane tiling by squares of diagonal length $\eta$. Let $S$ be the set of such squares $B$ satisfying $B \cap A \neq \emptyset$.

Recover each square of $S$ by a cover of cost less than $\tau+\varepsilon / 2$ and whose disks are of diameter less than $\eta$ (we did this in the preceding section).

The union of all such square covers of $S$ is a cover $\mathcal{P}$ of $A$, contained in $A(2 \eta)=\{x \in$ $\mathbb{R}^{2}$, dist $\left.(x, A)<2 \eta\right\}$.

If $A$ is a compact, $A(2 \eta)$ also is, and the total cost of $\mathcal{P}$ is less than $(\tau+\varepsilon / 2) \operatorname{Area}(A(2 \eta))$. Since $\lim _{\eta \rightarrow 0} \operatorname{Area}(A(2 \eta))=\operatorname{Area}(A)$ We can take $\eta$ such that

$$
\operatorname{Area}(A(2 \eta)) \leq \operatorname{Area}(A)\left(1+\frac{\varepsilon}{2(\tau+\varepsilon / 2)}\right) .
$$

With this choice, $\operatorname{Cost}(\mathcal{P}) \leq \operatorname{Area}(A)(\tau+\varepsilon)$, as wished.

If $A$ is not compact, the same idea still works, when considering the limit. 


\subsubsection{A plane cover with a cost equal to $\tau$}

This is the last point of the Theorem.

We first consider a connex cover $\mathcal{P}_{n}$ of the crown $D(O, n)-D(O, n-1)$ with a cost equal to $\left(1+\varepsilon / 2^{n}\right) \tau$. Then, we connect one disk of $\mathcal{P}_{n-1}$ to one disk of $\mathcal{P}_{n}$ by a little link $\mathcal{L}_{n}$ of cost inferior to $(1 /(2 n+1)) \tau$. The plane cover, reunion of all $\mathcal{P}_{n}$ and $\mathcal{L}_{n}$ is connex and its cost is inferior to:

$$
\begin{aligned}
\lim _{n} & \frac{\sum_{i=1}^{n}\left(\operatorname{Area}\left(D_{n}\right)-\operatorname{Area}\left(D_{n-1}\right)\right)\left(1+\frac{1}{(2 i+1)}\right)}{\operatorname{Area}\left(D_{n}\right)} \tau \\
& =\lim _{n \longrightarrow+\infty} \tau+\frac{\sum_{i=1}^{n} \pi(2 i+1) /(2 i+1)}{\pi n^{2}} \tau \\
& =\tau+\lim _{n \longrightarrow+\infty} \frac{n}{n^{2}} \tau \\
& =\tau .
\end{aligned}
$$

\section{Conclusion}

In this report, we have provided novel contributions and addressed several issues concerning the design of energy efficient broadcast and sensing algorithms for sensor networks. We have proposed a novel approach since it is the first time that both the transmission and the reception costs are taken into account in the evaluation of the energy consumption of a broadcasting task. We also gave a new analytical model for sensor networks where the use of a continuous distribution of nodes allows the derivation of upper and lower bounds on region covering. Based on this theory, we are able to prove that the derived lower bound is asymptotically optimal. This model also allowed us to propose energy efficient sensing and broadcast strategies and to develop a simple energy efficient broadcast algorithm.

For the future, possible extensions of this work are open to investigation. In order to closely follow the reality of sensor networks, we are currently investigating the integration of discrete levels of energy in our mathematical model. These levels would replace the continuous range of possible transmitting powers we are currently using. Once again, the challenge is to adapt our continuous theory to a pseudo discrete case.

\section{References}

[1] I. Akyildiz, W. Su, Y. Sankarasubramaniam, and E. Cayirci. A survey on sensor networks. IEEE Communications, pages 102-114, August 2002.

[2] L. Badia and M. Zorzi. On the construction of broadcast and multicast trees in wireless networks - global vs. local energy efficiency. In IEEE GLOBECOM2002, 2002. 
[3] S. Bandyopadhyay and E. Coyle. An energy efficient hierarchical clustering algorithm for wireless sensor networks. In INFOCOM 2003. IEEE, 2003.

[4] S. Banerjee and A. Misra. Energy-efficient broadcast and multicast trees for reliable wireless communication. In IEEE Wireless Communications and Networking Conference (WCNC), New Orleans, USA, March 2003.

[5] S. Bansal, R. Shorey, and A. Misra. Comparing the routing energy overheads of adhoc routing protocols. In IEEE Wireless Communications and Networking Conference (WCNC), New Orleans, USA, March 2003.

[6] K. Bult, A. Burstein, D. Chang, M. Dong, and W. Kaiser. Wireless integrated microsensors. In Proceedings of Conference on Sensors and Systems (Sensors Expo), pages 33-38, Anaheim, CA, April 1996.

[7] A. Clementi, P. Penna, and R. Silvestri. Hardness results for the power range assignment problem in packet radio networks. In International Workshop on Approximation Algirithms for Combinatorial Optimization (RANDOM/APPROX'99), volume LNCS (1671), pages 197-208, 1999.

[8] A. Clementi, P. Penna, and R. Silvestri. The power range assignment problem in packet radio networks on the plane. In Symposium on Theoretical Aspect of Computer Science (STACS'00), volume LNCS (1770), pages 651-660, 2000.

[9] D. Estrin, R. Govindan, J. Heidemann, and S. Kumar. Next century challenges: Scalable coordination in sensor networks. In Fifth Annual International Conference on Mobile Computing and Networks (MobiCOM '99), Seattle, Washington, August 1999.

[10] P. Fraigniaud and E. Lazard. Methods and problems of communication in usual networks. Discrete Applied Mathematics, 53:79-133, 1994. (special issue on broadcasting).

[11] W. Heinzelman, A. Chandrakasan, and H. Balakrishnan. Energy-efficient communication protocol for wireless microsensor networks. In 33rd Hawaii International Conference on System Sciences (HICSS '00), January 2000.

[12] J. Kahn, R. Katz, and K. Pister. Next century challenges: mobile networking for "smart dust". In MobiCom, 1999.

[13] K. Pahlavan and A. Levesque. Wireless Information Networks. Wiley Interscience, 1995. New-York.

[14] G. Pottie and L. Clare. Wireless integrated network sensors: toward low-cost and robust self-organizing security networks. In SPIE, Sensors, C3I, volume 3577, pages 86-95, 1998.

[15] V. Raghunathan, C. Schurgers, S. Park, and M. Srivastava. Energy-aware wireless microsensor networks. IEEE Signal Processing Magazine, 19(2), Mar 2002. 
[16] T. Rappaport. Wireless Communication, Principles and Practice. Prentice-Hall, 1996.

[17] C. Schurgers, V. Tsiatsis, S. Ganeriwal, and M. Srivastava. Optimizing sensor networks in the energy-latency-density design space. IEEE Transactions on Mobile Computing, 1(1):70-80, January-March 2002.

[18] E. Shih, S. Cho, N. Ickes, R. Min, A. Sinha, A. Wang, and A. Chandrakasan. Physical layer driven protocol and algorithm design for energy-efficient wireless sensor networks. In ACM MobiCom' 01, pages 272-286, Roma, Italy, July 2001.

[19] K. Sohrabi, J. Gao, V. Ailawadhi, and G. Pottie. Protocols for self-organization of a wireless sensor network. IEEE Personal Communication, pages 16-27, October 2000.

[20] F Tóth. Regular figures. In International Series of Monographs in Pure and Applied Mathematics. Pergamon Press, 1964.

[21] M. C̆agalj, J.-P. Hubaux, and C. Enz. Energy-efficient broadcasting in all-wireless networks. ACM Mobile Networks and Applications (MONET), 2003.

[22] P.-J. Wan, G. Călinescu, X.-Y. Li, and O. Frieder. Minimum-energy broadcasting in static ad hoc wireless network. Wireless Networks, 8:607-617, 2002.

[23] J. Wieselthier, G. Nguyen, and A. Ephremides. Energy-efficient broadcast and multicast trees in wireless networks. MONET, 7(6):481-492, 2002.

$\mathrm{RR} \mathrm{n}^{\circ} 5072$ 


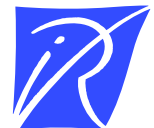

Unité de recherche INRIA Rhône-Alpes 655, avenue de l'Europe - 38334 Montbonnot Saint-Ismier (France)

Unité de recherche INRIA Futurs : Parc Club Orsay Université - ZAC des Vignes 4, rue Jacques Monod - 91893 ORSAY Cedex (France) Unité de recherche INRIA Lorraine : LORIA, Technopôle de Nancy-Brabois - Campus scientifique 615, rue du Jardin Botanique - BP 101 - 54602 Villers-lès-Nancy Cedex (France)

Unité de recherche INRIA Rennes : IRISA, Campus universitaire de Beaulieu - 35042 Rennes Cedex (France) Unité de recherche INRIA Rocquencourt : Domaine de Voluceau - Rocquencourt - BP 105 - 78153 Le Chesnay Cedex (France) Unité de recherche INRIA Sophia Antipolis : 2004, route des Lucioles - BP 93 - 06902 Sophia Antipolis Cedex (France) 